\title{
Effects of Hall Current on MHD Boundary Layer Second-Order Viscoelastic Fluid Flow Induced by a Continuous Surface with Heat Transfer
}

\author{
Haider Zaman, Murad Ali Shah, Farhan Khan, Qaiser Javed \\ Faculty of Numerical Sciences, Islamia College University, Peshawar, Pakistan \\ Email: haiderzaman67@yahoo.com \\ Received 31 January 2014; revised 25 February 2014; accepted 7 March 2014 \\ Copyright (C) 2014 by authors and Scientific Research Publishing Inc. \\ This work is licensed under the Creative Commons Attribution International License (CC BY). \\ http://creativecommons.org/licenses/by/4.0/

\begin{abstract}
Effects of Hall current on heat transfer and magnetohydrodynamic (MHD) boundary layer flow induced by a continuous surface in a parallel free stream of a second-order viscoelastic fluid are studied for uniform suction/injection by taking viscous dissipation into account. Complex nonsimilar solutions to the stream function and temperature are developed by means of an elegant technique, known as homotopy analysis method (HAM). Convergence of the solutions is ensured with the help of $\hbar$-curves. Graphical and tabular results for the effects of Hall current reveal that it has a significant influence on: complex velocity, complex temperature, magnitude of the shear stress at the surface, magnitude of the rate of heat transfer at the surface and on boundary layer thickness.
\end{abstract}

Keywords

Hall Currents, Heat Transfer, Boundary Layer, Complex Nonsimilar Solutions, HAM

\section{Introduction}

Most of the time Hall current was ignored in applying Ohm's law because it has no extraordinary effect for small and average values of the magnetic field. The effects of Hall current are very important in the presence of a strong magnetic field [1], because for strong magnetic field electromagnetic force is prominent. The recent research for the applications of MHD is towards a strong magnetic field, due to which study of Hall current is very important. Actually, in an ionized gas of low density subjected to a strong magnetic field, the conductivity perpendicular to the magnetic field is decreased by free spiral movement of electrons and ions about the magnetic lines of force 
before suffering collisions. A current produced in a direction at right angle to the electric and magnetic fields is called Hall current. The important engineering applications for MHD boundary layer flows with heat transfer including the effects of Hall current are encountered in MHD power generators and pumps, Hall accelerators, refrigeration coils, electric transformers, in flight MHD, solar physics involved in the sunspot development, the solar cycle, the structure of magnetic stars, electronic system cooling, cool combustors, fibre and granular insulation, oil extraction, thermal energy storage and flow through filtering devices and porous material regenerative heat exchangers. Some interesting studies regarding the effects of Hall current on MHD boundary layer flow are as follows:

Zaman [2] recently examined the effects of Hall current on the unsteady incompressible MHD fluid flow with slip conditions and porous walls. Ayub et al. [3] considered the effects of Hall current on hydromagnetic flow and heat transfer in a second grade fluid over a stretching sheet. The effects of Hall current on unsteady MHD flows of a second grade fluid are studied by Ahmad et al. [4]. Hall effects on hydromagnetic flow over a surface stretching with a power-law velocity and unsteady duct flow of a non-Newtonian fluid in a porous medium are investigated by Hayat et al. [5] [6]. The effects of Hall current and heat transfer on flow due to a pull of eccentric rotating disks are discussed by Asghar et al. [7]. Khan et al. [8] described the Hall effects on the pipe flow of a Burgers' fluid. Abo-Eldahab et al. [9] [10] analyzed the effects of Hall current on the mixed convection and free convection boundary layer flows of non-Newtonian and micropolar fluids. Debnath et al. [11] investigated the effects of Hall current on unsteady hydromagnetic flow past a porous plate in a rotating fluid system. The study of boundary layer flows from a continuous surface is important because it is involved in a large number of engineering processes. Since in the remarkable work of Sakiadis [12] such flows are studied extensively through various aspects, the literature on the topic is quite rich.

The present paper is devoted to study the effects of Hall current on heat transfer and MHD boundary layer flow induced by a continuous surface in a parallel free stream of a second-order viscoelastic fluid. The resulting Hall current problem is solved by means of homotopy analysis method (HAM) [13]-[19], which is very powerful and efficient in finding the analytic solutions for a wide class of nonlinear differential equations. The method gives more realistic analytic solution that converge very rapidly in physical problems. The convergence region for the complex nonsimilar series solution is found with the help of $\hbar$-curves. The effects of Hall current on the real and imaginary parts of velocity and temperature are seen for suction/injection with the help of graphs. Tables are constructed to discuss the effects of Hall current on magnitude of shear stress at the surface and rate of heat transfer at the surface for suction/injection.

\section{The Problem and Its Solution with Convergence Check}

The arising nonlinear problems for flow and heat transfer with effects of Hall current are [20] [21]

$$
\begin{gathered}
F^{\prime \prime \prime}+\frac{1}{2} F F^{\prime \prime}=\frac{K}{2}\left(F^{\prime \prime 2}-2 F^{\prime} F^{\prime \prime \prime}-F F^{i v}\right)+\frac{N(1+i \phi)}{\left(1+\phi^{2}\right)} F^{\prime}, \\
\Theta^{\prime \prime}+\frac{1}{2} P_{r} F \Theta^{\prime}=\frac{1}{2} P_{r} E K\left(F^{\prime} F^{\prime \prime 2}+F F^{\prime \prime} F^{\prime \prime \prime}\right)-P_{r} E F^{\prime \prime 2}, \\
F(0)=R, \quad F^{\prime}(0)=1-\lambda, \quad F^{\prime}(\infty)=\lambda, \\
\Theta(0)=1, \quad \Theta(\infty)=0 .
\end{gathered}
$$

where prime indicates the differentiation with respect to $\eta$ only, where $\eta$ is the distance from the surface, $\lambda\left(=U_{\infty} / U_{\infty}+U_{w}\right)$ is the velocity ratio, $R=-2 V_{0} \sqrt{x R_{e}}$ is the suction/injection parameter, $V_{0}$ is the suction/ injection velocity, $P_{r}(=v / \alpha)$ is the Prandtl number, $R_{e}$ is the Reynold number, $K\left(=\alpha_{1} U_{r} / \rho v x\right)$ is the second grade fluid parameter, $E\left(=U_{r}^{2} / c_{p}\left(T_{w}-T_{\infty}\right)\right)$ is the Eckert number, $\Theta$ is the dimensionless temperature, $N\left(=\sigma B_{0}^{2} L / \rho U_{r}\right)$ is magnetic parameter and $F$ is the dimensionless stream function, $R>0$ corresponds to suction and $R<0$ corresponds to injection. It is noticed that the nondimensional problems defined by above equations are nonsimilar. For analytic HAM solutions the initial approximations of $F(\eta)$ and $\Theta(\eta)$ are

$$
\begin{gathered}
F_{0}(\eta)=(1+R-2 \lambda)+\lambda \eta-(1-2 \lambda) \mathrm{e}^{-\eta}, \\
\Theta_{0}(\eta)=\mathrm{e}^{-\eta},
\end{gathered}
$$


and the auxiliary linear operators are

$$
\begin{aligned}
& \mathcal{L}_{1}(F)=F^{\prime \prime \prime}-F^{\prime}, \\
& \mathcal{L}_{2}(\Theta)=\Theta^{\prime \prime}-\Theta,
\end{aligned}
$$

Following the HAM and trying higher iterations with the unique and proper assignment of the results converge to the exact solution:

$$
\begin{gathered}
F(\eta) \approx F_{0}(\eta)+F_{1}(\eta)+F_{2}(\eta)+\cdots+F_{m}(\eta), \\
\Theta(\eta) \approx \Theta_{0}(\eta)+\Theta_{1}(\eta)+\Theta_{2}(\eta)+\cdots+\Theta_{m}(\eta),
\end{gathered}
$$

using the symbolic computation software such as MATLAB, MAPLE or MATHEMATICA successively obtain

$$
\begin{aligned}
& F_{1}(\eta)=1-\frac{i \hbar_{1}}{3(i+\phi)}+\frac{i K \hbar_{1}}{12(i+\phi)}+\frac{i N \hbar_{1}}{2(i+\phi)}-\frac{\hbar_{1} \phi}{3(i+\phi)}+\frac{K \hbar_{1} \phi}{12(i+\phi)}+R \\
& +\frac{i K R \hbar_{1}}{4(i+\phi)}+\frac{\phi R \hbar_{1}}{4(i+\phi)}+\frac{K \phi R \hbar_{1}}{4(i+\phi)}-2 \lambda+\frac{17 i R \hbar_{1} \lambda}{24(i+\phi)}-\frac{11 i K \hbar_{1} \lambda}{24(i+\phi)} \\
& -\frac{2 i N \hbar_{1} \lambda}{(i+\phi)}+\frac{17 \phi \hbar_{1} \lambda}{24(i+\phi)}-\frac{11 K \phi \hbar_{1} \lambda}{24(i+\phi)}-\frac{i R \hbar_{1} \lambda}{2(i+\phi)}-\frac{i K R \hbar_{1} \lambda}{2(i+\phi)}-\frac{R \phi \hbar_{1} \lambda}{2(i+\phi)} \\
& +\eta \lambda+\frac{i N \phi \hbar_{1} \eta \lambda}{(i+\phi)}-\frac{i \hbar_{1} \lambda^{2}}{12(i+\phi)}+\frac{7 i K \hbar_{1} \lambda^{2}}{12(i+\phi)}-\frac{\hbar_{1} \phi \lambda^{2}}{12(i+\phi)}+\frac{7 K \hbar_{1} \phi \lambda^{2}}{12(i+\phi)} \\
& -\frac{i \hbar_{1} \mathrm{e}^{-2 \eta}}{12(i+\phi)}-\frac{i K \hbar_{1} \mathrm{e}^{-2 \eta}}{6(i+\phi)}-\frac{\hbar_{1} \phi \mathrm{e}^{-2 \eta}}{12(i+\phi)}-\frac{K \hbar_{1} \phi \mathrm{e}^{-2 \eta}}{6(i+\phi)}+\frac{i \hbar_{1} \lambda \mathrm{e}^{-2 \eta}}{3(i+\phi)}+\frac{2 i K \hbar_{1} \lambda \mathrm{e}^{-2 \eta}}{3(i+\phi)} \\
& +\frac{\hbar_{1} \phi \lambda \mathrm{e}^{-2 \eta}}{3(i+\phi)}+\frac{2 K \hbar_{1} \phi \lambda \mathrm{e}^{-2 \eta}}{3(i+\phi)}-\frac{i \hbar_{1} \lambda^{2} \mathrm{e}^{-2 \eta}}{3(i+\phi)}-\frac{2 i K \hbar_{1} \lambda^{2} \mathrm{e}^{-2 \eta}}{3(i+\phi)}-\frac{\hbar_{1} \phi \lambda^{2} \mathrm{e}^{-2 \eta}}{3(i+\phi)} \\
& -\frac{2 K \hbar_{1} \phi \lambda^{2} \mathrm{e}^{-2 \eta}}{3(i+\phi)}-\mathrm{e}^{-\eta}+\frac{5 i \hbar_{1} \mathrm{e}^{-\eta}}{12(i+\phi)}+\frac{i K \hbar_{1} \mathrm{e}^{-\eta}}{12(i+\phi)}-\frac{i \hbar_{1} N \mathrm{e}^{-\eta}}{2(i+\phi)}+\frac{5 \hbar_{1} \phi \mathrm{e}^{-\eta}}{12(i+\phi)} \\
& +\frac{K \hbar_{1} \phi \mathrm{e}^{-\eta}}{12(i+\phi)}-\frac{i R \hbar_{1} \mathrm{e}^{-\eta}}{4(i+\phi)}-\frac{i K R \hbar_{1} \mathrm{e}^{-\eta}}{4(i+\phi)}-\frac{R \hbar_{1} \phi \mathrm{e}^{-\eta}}{4(i+\phi)}-\frac{K R \hbar_{1} \phi \mathrm{e}^{-\eta}}{4(i+\phi)}+\frac{i \hbar_{1} \eta \mathrm{e}^{-\eta}}{4(i+\phi)} \\
& -\frac{i K \hbar_{1} \eta \mathrm{e}^{-\eta}}{4(i+\phi)}-\frac{i N \hbar_{1} \eta \mathrm{e}^{-\eta}}{2(i+\phi)}+\frac{\hbar_{1} \phi \eta \mathrm{e}^{-\eta}}{4(i+\phi)}-\frac{\phi K \hbar_{1} \eta \mathrm{e}^{-\eta}}{4(i+\phi)}-\frac{i R \hbar_{1} \eta \mathrm{e}^{-\eta}}{4(i+\phi)} \\
& -\frac{i K R \hbar_{1} \eta \mathrm{e}^{-\eta}}{4(i+\phi)}-\frac{\phi R \hbar_{1} \eta \mathrm{e}^{-\eta}}{4(i+\phi)}-\frac{K \phi R \hbar_{1} \eta \mathrm{e}^{-\eta}}{4(i+\phi)}+2 \lambda-\frac{25 i \hbar_{1} \lambda \mathrm{e}^{-\eta}}{24(i+\phi)}-\frac{5 i \hbar_{1} K \lambda \mathrm{e}^{-\eta}}{24(i+\phi)} \\
& +\frac{2 i \hbar_{1} N \lambda \mathrm{e}^{-\eta}}{(i+\phi)}-\frac{25 \hbar_{1} \phi \lambda \mathrm{e}^{-\eta}}{24(i+\phi)}-\frac{5 K \hbar_{1} \phi \lambda \mathrm{e}^{-\eta}}{24(i+\phi)}+\frac{i \hbar_{1} R \lambda \mathrm{e}^{-\eta}}{2(i+\phi)}+\frac{i K \hbar_{1} R \lambda \mathrm{e}^{-\eta}}{2(i+\phi)} \\
& +\frac{\hbar_{1} \phi R \lambda \mathrm{e}^{-\eta}}{2(i+\phi)}+\frac{K \hbar_{1} \phi R \lambda \mathrm{e}^{-\eta}}{2(i+\phi)}-\frac{3 i \hbar_{1} \eta \lambda \mathrm{e}^{-\eta}}{8(i+\phi)}+\frac{9 i K \hbar_{1} \eta \lambda \mathrm{e}^{-\eta}}{8(i+\phi)}+\frac{i N \hbar_{1} \eta \lambda \mathrm{e}^{-\eta}}{(i+\phi)} \\
& -\frac{3 \phi \hbar_{1} \eta \lambda \mathrm{e}^{-\eta}}{8(i+\phi)}+\frac{9 K \phi \hbar_{1} \eta \lambda \mathrm{e}^{-\eta}}{8(i+\phi)}+\frac{i \hbar_{1} R \eta \lambda \mathrm{e}^{-\eta}}{2(i+\phi)}+\frac{i K \hbar_{1} R \eta \lambda \mathrm{e}^{-\eta}}{2(i+\phi)}+\frac{\phi \hbar_{1} R \eta \lambda \mathrm{e}^{-\eta}}{2(i+\phi)} \\
& +\frac{K \phi \hbar_{1} R \eta \lambda \mathrm{e}^{-\eta}}{2(i+\phi)}-\frac{i \hbar_{1} \eta^{2} \lambda \mathrm{e}^{-\eta}}{8(i+\phi)}-\frac{i K \hbar_{1} \eta^{2} \lambda \mathrm{e}^{-\eta}}{8(i+\phi)}-\frac{\phi \hbar_{1} \eta^{2} \lambda \mathrm{e}^{-\eta}}{8(i+\phi)}-\frac{K \phi \hbar_{1} \eta^{2} \lambda \mathrm{e}^{-\eta}}{8(i+\phi)} \\
& +\frac{5 i \hbar_{1} \lambda^{2} \mathrm{e}^{-\eta}}{12(i+\phi)}+\frac{i K \hbar_{1} \lambda^{2} \mathrm{e}^{-\eta}}{12(i+\phi)}+\frac{5 \hbar_{1} \phi \lambda^{2} \mathrm{e}^{-\eta}}{12(i+\phi)}+\frac{K \hbar_{1} \phi \lambda^{2} \mathrm{e}^{-\eta}}{12(i+\phi)}-\frac{i \hbar_{1} \eta \lambda^{2} \mathrm{e}^{-\eta}}{4(i+\phi)} \\
& -\frac{5 i K \hbar_{1} \eta \lambda^{2} \mathrm{e}^{-\eta}}{4(i+\phi)}-\frac{\hbar_{1} \phi \eta \lambda^{2} e^{-\eta}}{4(i+\phi)}-\frac{5 K \hbar_{1} \phi \eta \lambda^{2} \mathrm{e}^{-\eta}}{4(i+\phi)}+\frac{i \hbar_{1} \eta^{2} \lambda^{2} \mathrm{e}^{-\eta}}{4(i+\phi)}+\frac{i K \hbar_{1} \eta^{2} \lambda^{2} \mathrm{e}^{-\eta}}{4(i+\phi)} \\
& +\frac{\hbar_{1} \phi \eta^{2} \lambda^{2} \mathrm{e}^{-\eta}}{4(i+\phi)}+\frac{K \hbar_{1} \phi \eta^{2} \lambda^{2} \mathrm{e}^{-\eta}}{4(i+\phi)}
\end{aligned}
$$




$$
\begin{aligned}
\Theta_{1}(\eta)= & \mathrm{e}^{-\eta}+\frac{1}{6} \mathrm{e}^{-2 \eta} \hbar_{2}-\frac{1}{6} \mathrm{e}^{-\eta} \hbar_{2} P_{r}-\frac{1}{2} \mathrm{e}^{-\eta} \hbar_{2} \eta+\frac{1}{4} \mathrm{e}^{-\eta} \hbar_{2} P_{r} \eta+\frac{1}{4} \mathrm{e}^{-\eta} \hbar_{2} P_{r} R \eta \\
& -\frac{1}{3} \mathrm{e}^{-2 \eta} \hbar_{2} P_{r} \lambda+\frac{1}{3} \mathrm{e}^{-\eta} \hbar_{2} P_{r} \lambda-\frac{3}{8} \mathrm{e}^{-\eta} \hbar_{2} P_{r} \eta+\frac{1}{8} \mathrm{e}^{-\eta} \hbar_{2} P_{r} \eta^{2} \lambda-\frac{1}{8} K \mathrm{e}^{-3 \eta} \hbar_{2} P_{r} E \\
& +\frac{1}{3} \mathrm{e}^{-2 \eta} \hbar_{2} P_{r} E+\frac{1}{6} K \mathrm{e}^{-2 \eta} \hbar_{2} P_{r} E-\frac{1}{3} \mathrm{e}^{-\eta} \hbar_{2} P_{r} E-\frac{1}{24} K \mathrm{e}^{-\eta} \hbar_{2} P_{r} E+\frac{1}{6} K \mathrm{e}^{-2 \eta} \hbar_{2} P_{r} R E \\
& -\frac{1}{6} K \mathrm{e}^{-\eta} \hbar_{2} P_{r} R E+\frac{3}{4} K \mathrm{e}^{-3 \eta} \hbar_{2} P_{r} \lambda E-\frac{4}{3} \mathrm{e}^{-2 \eta} \hbar_{2} P_{r} \lambda E-\frac{17}{18} K \mathrm{e}^{-2 \eta} \hbar_{2} P_{r} \lambda E \\
& +\frac{4}{3} \mathrm{e}^{-\eta} \hbar_{2} P_{r} \lambda E+\frac{7}{36} K \mathrm{e}^{-\eta} \hbar_{2} P_{r} \lambda E-\frac{2}{3} K \mathrm{e}^{-2 \eta} \hbar_{2} P_{r} R \lambda E+\frac{2}{3} K \mathrm{e}^{-\eta} \hbar_{2} P_{r} R \lambda E \\
& +\frac{1}{6} K \mathrm{e}^{-2 \eta} \hbar_{2} P_{r} \eta \lambda E-\frac{3}{2} K \mathrm{e}^{-3 \eta} \hbar_{2} P_{r} \lambda^{2} E+\frac{4}{3} \mathrm{e}^{-2 \eta} \hbar_{2} P_{r} \lambda^{2} E+\frac{16}{9} K \mathrm{e}^{-2 \eta} \hbar_{2} P_{r} \lambda^{2} E \\
& -\frac{4}{3} \mathrm{e}^{-\eta} \hbar_{2} P_{r} \lambda^{2} E-\frac{5}{18} K \mathrm{e}^{-\eta} \hbar_{2} P_{r} \lambda^{2} E+\frac{2}{3} K \mathrm{e}^{-2 \eta} \hbar_{2} P_{r} R \lambda^{2} E-\frac{2}{3} K \mathrm{e}^{-\eta} \hbar_{2} P_{r} R \lambda^{2} E \\
& -\frac{2}{3} K \mathrm{e}^{-2 \eta} \hbar_{2} P_{r} \eta \lambda^{2} E+K \mathrm{e}^{-3 \eta} \hbar_{2} P_{r} \lambda^{3} E-\frac{10}{9} K \mathrm{e}^{-2 \eta} \hbar_{2} P_{r} \lambda^{3} E \\
& +\frac{1}{9} K \mathrm{e}^{-\eta} \hbar_{2} P_{r} \lambda^{3} E+\frac{2}{3} K \mathrm{e}^{-2 \eta} \hbar_{2} P_{r} \eta \lambda^{3} E
\end{aligned}
$$

The complete complex nonsimilar analytic solutions are

$$
\begin{array}{r}
F(\eta)=\sum_{m=0}^{\infty} F_{m}(\eta)=\lim _{M \rightarrow \infty}\left[\sum_{m=0}^{M}\left(\sum_{k=0}^{2 m+1} \Gamma_{m, 0}^{k} \eta^{k}\right)+\sum_{n=1}^{M+1} \mathrm{e}^{-n \eta}\left(\sum_{m=n-1}^{M} \sum_{k=0}^{2 m+1-n} \Gamma_{m, n}^{k} \eta^{k}\right)\right], \\
\Theta(\eta)=\sum_{m=0}^{\infty} \Theta_{m}(\eta)=\lim _{M \rightarrow \infty}\left[\sum_{n=1}^{M+1} \mathrm{e}^{-n \eta}\left(\sum_{m=n-1}^{M} \sum_{k=0}^{2 m+1-n} \Delta_{m, n}^{k} \eta^{k}\right)\right] .
\end{array}
$$

The coefficients $\Gamma_{m, n}^{k}$ and $\Delta_{m, n}^{k}$ can be computed by using

$$
\begin{gathered}
\Gamma_{0,0}^{0}=1+R-2 \lambda, \quad \Gamma_{0,1}^{0}=\lambda, \quad \Gamma_{0,0}^{1}=-1+2 \lambda, \\
\Delta_{0,0}^{0}=0, \quad \Delta_{0,0}^{1}=0, \quad \Delta_{0,1}^{0}=1 .
\end{gathered}
$$

given by the initial guess approximations in Equations (5) and (6).

We know that convergence region and rate of approximation for the homotopy analysis method (HAM) depend upon homotopy parameters $\hbar_{1}$ and $\hbar_{2}$. Therefore the $\hbar$-curves are sketched for $\operatorname{Re}(F(\eta))$ and $\operatorname{Re}(\Theta(\eta))$. Figure 1 and Figure 2 show that the range for the admissible values of $\hbar_{1}$ and $\hbar_{2}$ are $-1.5<\hbar_{1}<-0.5$ and $-1.5<\hbar_{2}<-0.5$, respectively. The computations made show that the series of the dimensionless stream function in Equation (13) converges in the whole region of $\eta$ when $\hbar_{1}=-0.8$ and series (14) converges in the whole region of $\eta$ when $\hbar_{1}=-0.8$ and $\hbar_{2}=-0.6$.

\section{Graphs, Tables and Discussion}

In order to discuss the effects of Hall current $\phi$ on the flow field the graphs are drawn for the variation of the real and imaginary parts of the velocity profile $F^{\prime}(\eta)$ with distance from the surface $\eta$ and for the real and imaginary parts of the shear stress at the wall for different values of the suction/injection parameter $R$, velocity ratio $\lambda$, second grade fluid parameter $K$, magnetic field parameter $N$ and the homotopy parameter $\hbar_{1}$ at constant wall temperature.

Figure 3 and Figure 4 elucidate the variation of the real and imaginary parts of the velocity profile $F^{\prime}(\eta)$ with $\eta$ for suction respectively. Figure 3 shows that for fixed values of $\hbar_{1}, N, K, R$ and $\lambda$ with increase in Hall current $\phi$ real part of the velocity increases for suction. Figure 4 describes that for fixed values of $\hbar_{1}, N, K, R$ and $\lambda$ with increase in Hall current $\phi$ imaginary part of the velocity increases in magnitude for suction. Similar behavior is observed for injection. In Figure 3 and Figure 4 boundary layer structure is observed and the boundary layer thickness increases with increasing Hall current $\phi$, which results in thick- 


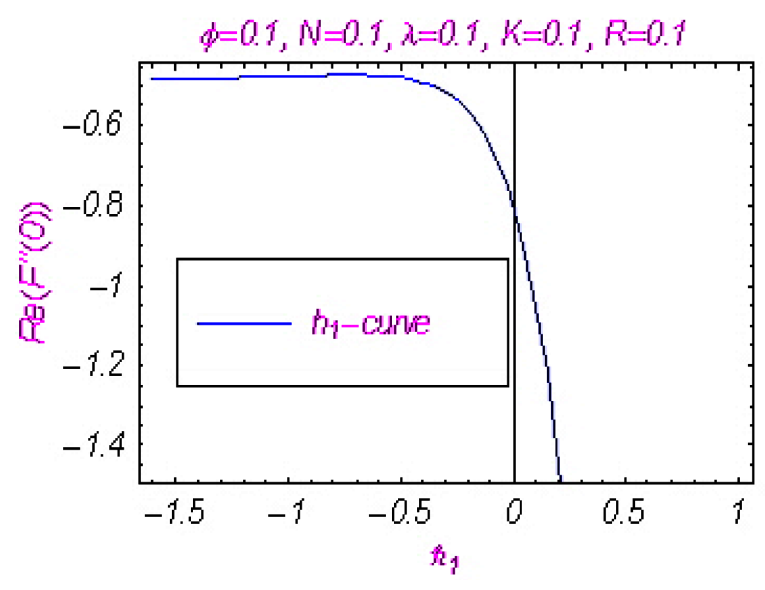

Figure 1. $\hbar_{1}$-curve for $\operatorname{Re}(F(\eta))$.

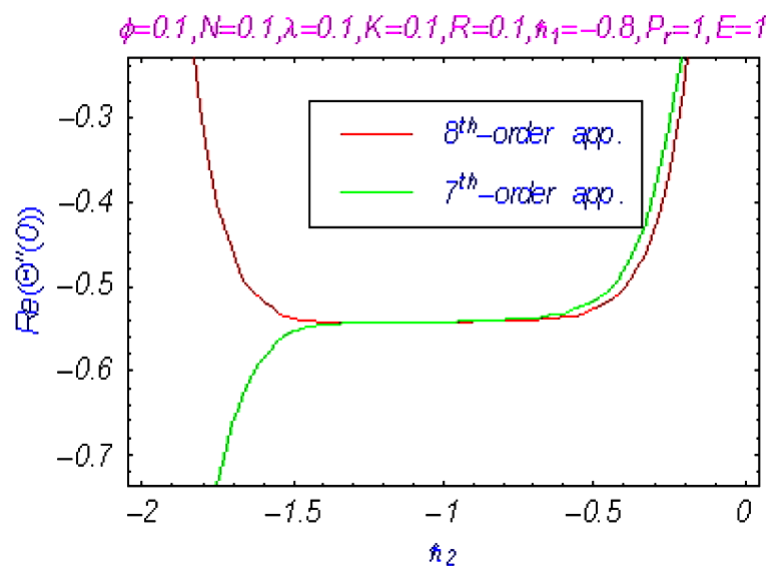

Figure 2. $\hbar_{2}$-curve for $\operatorname{Re}(\Theta(\eta))$.

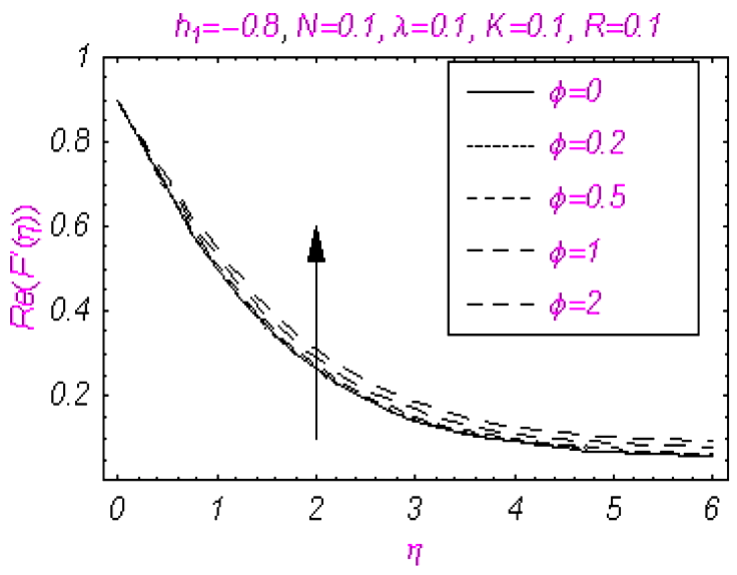

Figure 3. Influence of $\phi$ on $\operatorname{Re}\left(F^{\prime}(\eta)\right)$.

ening of the boundary layer. Hence, as expected, the velocity increases with increasing Hall current $\phi$. This is due to the fact that the effective conductivity decreases with increasing Hall current $\phi$, which reduces the magnetic damping force on the velocity. Figure 5 shows the variation of the real part of the skin friction coefficient 
$F^{\prime \prime}(0)$ with $N$ for several values of Hall current $\phi$ for suction. It is found that with increasing Hall current $\phi$ the skin friction coefficient decreases at all points for suction. Figure 6 indicates the variation of the real part of the skin friction coefficient $F^{\prime \prime}(0)$ with suction / injection parameter $R$ for several values of Hall current $\phi$. From Figure 6 it is seen that with increase in Hall current $\phi$ real part of the skin friction coefficient $F^{\prime \prime}(0)$ decreases. When magnetic field is applied perpendicular to the fluid velocity then it gives rise to a drag-like or resistive force which slow down or suppress the motion of the fluid on the surface. This leads to a reduction in the velocity of the fluid and flow rates. With the increase in the strength of the magnetic field the motion of the particulate suspension on the surface reduces due to which skin friction coefficient reduces as in Figure 5 and Figure 6.

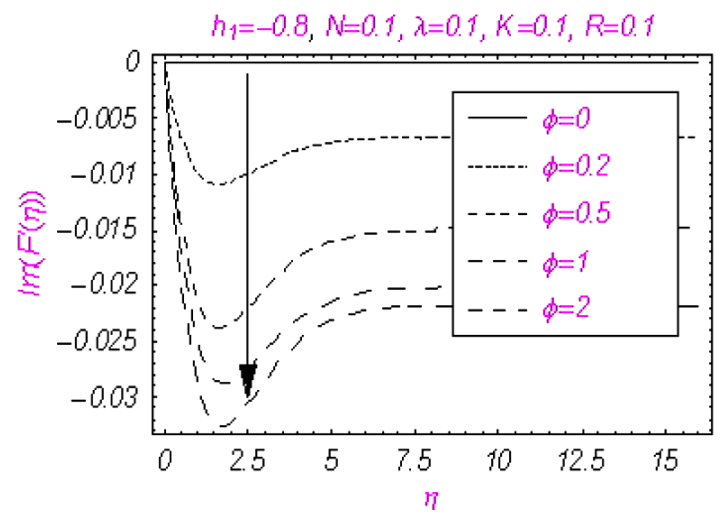

Figure 4. Influence of $\phi$ on $\operatorname{Im}\left(F^{\prime}(\eta)\right)$.

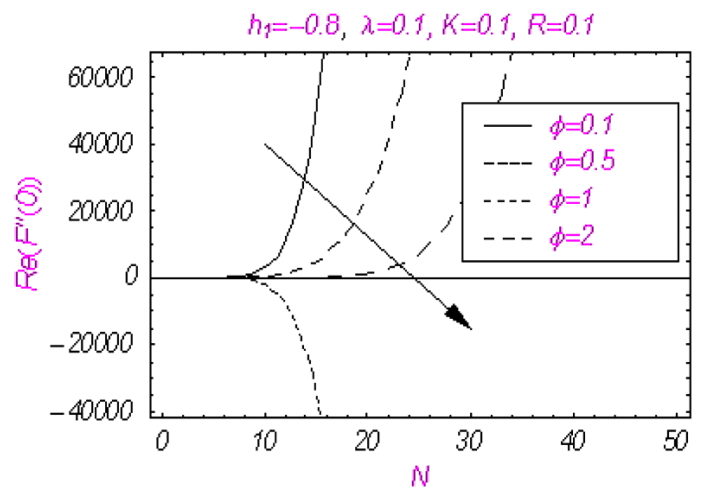

Figure 5. Influence of $\phi$ on $\operatorname{Re}\left(F^{\prime \prime}(0)\right)$.

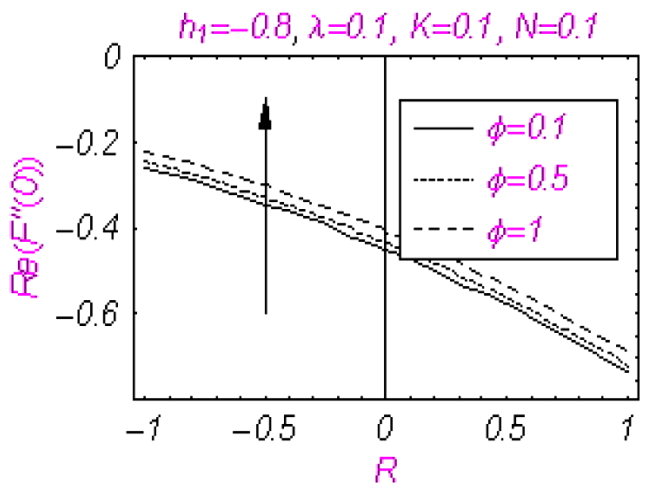

Figure 6. Influence of $\phi$ on $\operatorname{Re}\left(F^{\prime \prime}(0)\right)$. 
In order to discuss the effects of Hall current $\phi$ on the temperature field $\Theta(\eta)$ the graphs are plotted for different order of approximations for the variation of the real and imaginary parts of $\Theta(\eta)$ with distance from the surface $\eta$ and for the real and imaginary parts of the local Nusselt number $\Theta^{\prime}(0)$ for different values of the seven dimensionless parameters (i) suction/injection parameter $R$ (ii) velocity ratio $\lambda$ (iii) elastic parameter $K$ (iv) magnetic field parameter $N$ (v) homotopy parameters $\hbar_{1}, \hbar_{2}$ (vi) Prandtl number $P_{r}$ and (vii) Eckert number $E$.

Figure 7 and Figure 8 depict the variation of the real and imaginary parts of the temperature profile $\Theta(\eta)$ with $\eta$ for several values of the Hall current $\phi$ for suction. It is observed from Figure 7 that for fixed values of $\hbar_{1}, \hbar_{2}, P_{r}, N, K, E, R$ and $\lambda$ with increase in Hall current $\phi$ real part of the temperature distribution increases for suction. Figure 8 shows that imaginary part of the temperature $\Theta(\eta)$ increases by increasing $\phi$. Same type of behavior is observed for injection. Figure 9 explains that for fixed values of $\hbar_{1}, \hbar_{2}, N, K, E$, $R$ and $\lambda$ with increase in Hall current $\phi$ real part of the temperature gradient $\Theta^{\prime}(0)$ decreases at all points for suction and decreases for injection for all values of the Prandtl number $P_{r}$. Figure 10 elucidates that imaginary part of the temperature gradient $\Theta^{\prime}(0)$ also decreases with increasing Hall current $\phi$.

Table 1 shows the absolute values of skin friction coefficient $F^{\prime \prime}(0)$ for Hall parameter, $\phi=0.0,0.1,0.3,0.7,1.0, \quad \hbar_{1}=-0.8$. It is observed from Table 1 that a decrease in $R$ leads to a reduction in the values of $F^{\prime \prime}(0)$. It is also observed from Table 1 that for a fixed value of $R$ with increase in Hall current $\phi$ magnitude of shear stress at wall decreases. Table 2 indicates the absolute values of heat transfer rate $\Theta^{\prime}(0)$ for

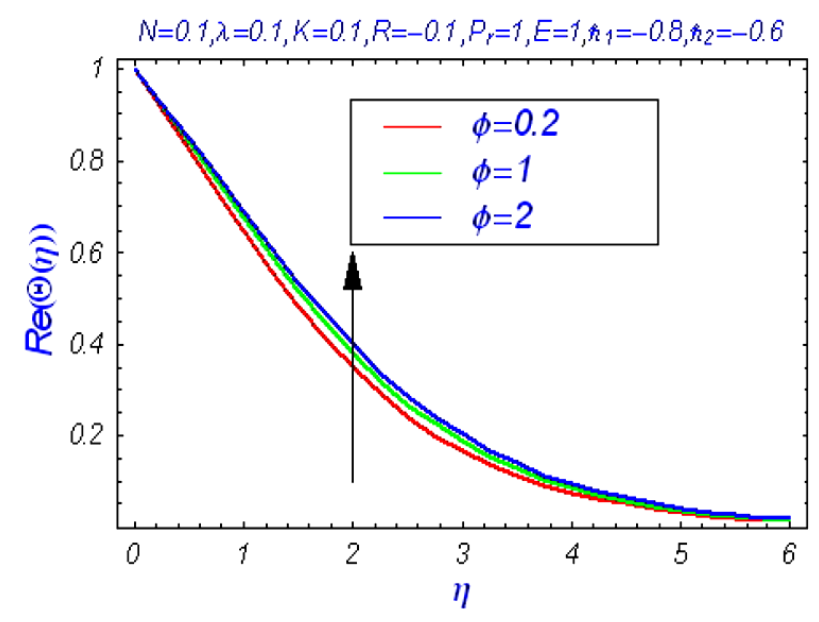

Figure 7. Influence of $\phi$ on $\operatorname{Re}(\Theta(\eta))$.

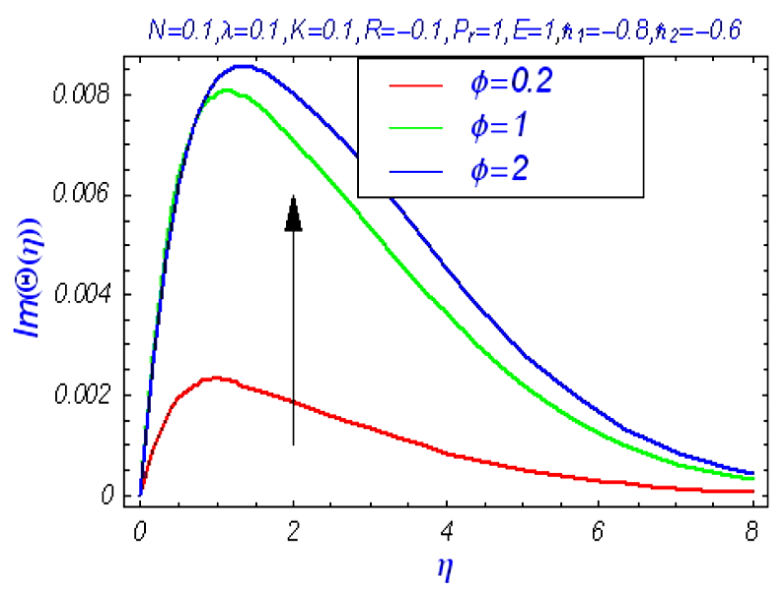

Figure 8. Influence of $\phi$ on $\operatorname{Im}(\Theta(\eta))$. 


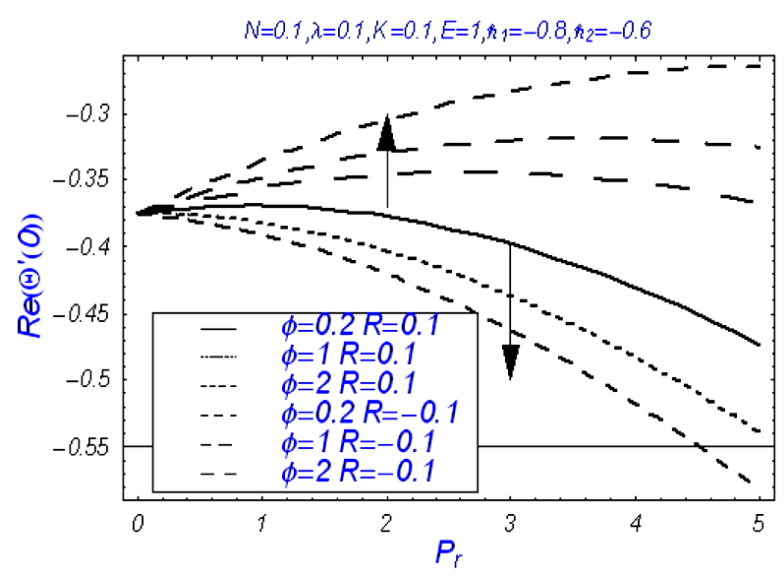

Figure 9. Influence of $\phi$ on $\operatorname{Re}\left(\Theta^{\prime}(0)\right)$.

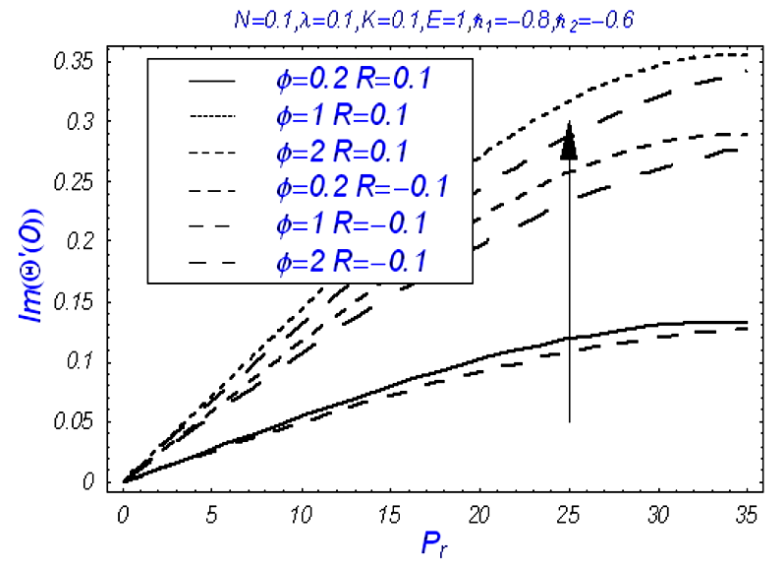

Figure 10. Influence of $\phi$ on $\operatorname{Im}\left(\Theta^{\prime}(0)\right)$.

Table 1. Absolute Values of skin friction coefficient $F^{\prime \prime}(0)$ with $K=0.1, \quad \lambda=0.1, \quad N=0.1, \quad \hbar_{1}=-0.8$.

$\begin{array}{cccccc}R & \phi=0.0 & \phi=0.1 & \phi=0.3 & \phi=0.7 & \phi=1.0 \\ 1 & 0.74722 & 0.74644 & 0.74055 & 0.71861 & 0.70113 \\ 0.5 & 0.58678 & 0.58390 & 0.58037 & 0.55929 & 0.54255 \\ 0 & 0.45300 & 0.45228 & 0.44684 & 0.42521 & 0.41049 \\ -0.5 & 0.34319 & 0.34250 & 0.33736 & 0.31814 & 0.30277 \\ -1 & 0.25544 & 0.25481 & 0.25006 & 0.23210 & 0.21784\end{array}$

$P_{r}=0.1, \quad \phi=0.0,0.1,0.3,0.7,1.0, \quad \hbar_{1}=-0.8, \quad \hbar_{2}=-0.6$. We observed from Table 2 that for a fixed value of Hall current $\phi$ a decrease in the values of $R$ yields reduction in $\Theta^{\prime}(0)$. Also for a fixed value of $R$ increase in Hall current $\phi$ leads to a reduction in local Nusselt number $\Theta^{\prime}(0)$. It can be seen from Table 3 that for fixed values of $R, K, \lambda, \quad N, P_{r}, \hbar_{1}, \hbar_{2}$ and $E$ the magnitude of $\Theta^{\prime}(0)$ for suction decreases with an increase in Hall current $\phi$ and for injection increases with an increase in $\phi$. Also for fixed values of $R, K, \lambda, N$, $P_{r}, \hbar_{1}, \hbar_{2}$ and $\phi$ the magnitude of $\Theta^{\prime}(0)$ (for suction/injection) decreases by increasing Eckert number E. 
Table 2. Absolute Values of heat transfer coefficient $\Theta^{\prime}(0)$ when $P_{r}=0.1, \quad E=2, \lambda=0.1, \quad N=0.1, \quad K=0.1$, $\hbar_{1}=-0.8, \quad \hbar_{2}=-0.6$.

\begin{tabular}{cccccc}
\hline$R$ & $\phi=0.0$ & $\phi=0.1$ & $\phi=0.3$ & $\phi=0.7$ & $\phi=1.0$ \\
1 & 0.349610 & 0.349588 & 0.349508 & 0.349476 & 0.349470 \\
0.5 & 0.324484 & 0.324456 & 0.324239 & 0.323382 & 0.322661 \\
0 & 0.286870 & 0.286778 & 0.286114 & 0.283606 & 0.281610 \\
-0.5 & 0.230163 & 0.229986 & 0.228659 & 0.223712 & 0.219829 \\
-1 & 0.146900 & 0.146619 & 0.144518 & 0.136621 & 0.130320 \\
\hline
\end{tabular}

Table 3. Absolute Values of temperature gradient at the wall $\Theta^{\prime}(0)$ at $P_{r}=0.1, \lambda=0.1, \quad N=0.1, \quad K=0.1, \quad \hbar_{1}=-0.8$, $\hbar_{2}=-0.6$.

\begin{tabular}{ccccc}
\hline $\mathrm{R}$ & $\phi$ & $E=2$ & $E=3$ & $E=4$ \\
\hline-1 & 0 & 0.1469 & 0.088422 & 0.033348 \\
& 1 & 0.130317 & 0.07007 & 0.03312 \\
& 2 & 0.1172 & 0.053389 & 0.032754 \\
& 3 & 0.112094 & 0.045901 & 0.031674 \\
& 4 & 0.109862 & 0.042334 & 0.029945 \\
& 5 & 0.108728 & 0.04043 & 0.26077 \\
& 0 & 0.29484 & 0.2778 & 0.262491 \\
& 1 & 0.295456 & 0.279972 & 0.264137 \\
& 2 & 0.29611 & 0.280122 & 0.264799 \\
& 3 & 0.296379 & 0.280589 & 0.265089 \\
& 4 & 0.296498 & 0.280793 & 0.265236 \\
\hline
\end{tabular}

\section{Conclusions}

In this study the Complex nonsimilar HAM solutions for the stream function and temperature are constructed on taking viscous dissipation and Hall currents into account. More significantly, the complex nonsimilar series solution clearly demonstrates how various physical parameters play their part in determining properties of the flow. The results are discussed under the effects of parameter $R, K, \lambda, N, P_{r}, E, \hbar_{1}$ and $\hbar_{2}$ through graphs and tables. We have following observations:

- The Complex solution series for dimensionless stream function $F(\eta)$ and dimensionless temperature function $\Theta(\eta)$ converges in the whole region of $\eta$ for $-1.5<\hbar_{1}<-0.5$ and $-1.5<\hbar_{2}<-0.5$.

- Real and imaginary parts of the fluid velocity increases with increase in Hall current $\phi$ for suction/injection.

- Real and imaginary parts of the fluid temperature increases with increase in Hall current $\phi$ for suction/injection.

- Boundary layer thickness increases for suction/injection with increasing Hall current $\phi$, which results in thickening of the boundary layer.

- Absolute values of the skin friction coefficient decreases with increase in Hall current $\phi$ for suction/injection.

- Increase in Hall current $\phi$ leads to a reduction in the absolute values of the local Nusselt number. 


\section{References}

[1] Cramer, K. and Pai, S. (1973) Magnetofluid Dynamics for Engineers and Applied Physicists. McGraw-Hill, New York.

[2] Zaman, H. (2013) Hall Effects on the Unsteady Incompressible MHD Fluid Flow with Slip Conditions and Porous Walls. Applied Mathematics and Physics, 1, 31-38.

[3] Ayub, M., Zaman, H. and Ahmad, M. (2010) Series Solution of Hydromagnetic Flow and Heat Transfer with Hall Effect in a Second Grade Fluid over a Stretching Sheet. Central European Journal of Physics, 8, 135-149. http://dx.doi.org/10.2478/s11534-009-0110-0

[4] Ahmad, M., Zaman, H. and Rehman, N. (2010) Effects of Hall Current on Unsteady MHD Flows of a Second Grade Fluid. Central European Journal of Physics, 8, 422-431. http://dx.doi.org/10.2478/s11534-009-0083-z

[5] Hayat, T., Zaman, H. and Ayub, M., (2010) Analytic Solution of Hydromagnetic Flow with Hall Effect over a Surface Stretching with a Power Law Velocity. Numerical Methods for Partial Differential Equations, 27, 937-959. http://dx.doi.org/10.1002/num.20562

[6] Hayat, T., Naz, R. and Asghar, S. (2004) Hall Effects on Unsteady Duct Flow of a Non-Newtonian Fluid in a Porous Medium. Applied Mathematics and Computation, 157, 103-114. http://dx.doi.org/10.1016/j.amc.2003.08.069

[7] Asghar, S., Mohyuddin R.M. and Hayat, T. (2005) Effects of Hall Current and Heat Transfer on Flow Due to a Pull of Ecentric Rotating Disks. International Journal of Heat and Mass Transfer, 48, 599-607. http://dx.doi.org/10.1016/j.ijheatmasstransfer.2004.08.023

[8] Khan, M., Asghar S. and Hayat, T. (2009) Hall Effect on the Pipe Flow of a Burgers' Fluid: An Exact Solution. Nonlinear Analysis: Real World Applications, 10, 974-979. http://dx.doi.org/10.1016/j.nonrwa.2007.11.016

[9] Abo-Eldahab, E.M. and Elbarbary, M.E. (2001) Hall Current Effect on Magnetohydrodynamic Free Convection Flow past a Semi-Infinite Vertical Plate with Mass Transfer. International Journal of Engineering Science, 39, 1641-1652. http://dx.doi.org/10.1016/S0020-7225(01)00020-9

[10] Abo-Eldahab, E.M. and Abd El Aziz, M. (2004) Hall Current and Ohmic Heating Effects on Mixed Convection Boundary Layer Flow of a Micropolar Fluid from a Rotating Cone with Power Law Variation in Surface Temperature. International Communications in Heat and Mass Transfer, 31, 751-762. http://dx.doi.org/10.1016/S0735-1933(04)00062-4

[11] Debnath, L., Ray, S.C. and Chatterjee, A.K. (1979) Effects of Hall Current on Unsteady Hydromagnetic Flow past a Porous Plate in a Rotating Fluid System. ZAMM-Zeitschrift für Angewandte Mathematik und Mechanik, 59, 469-471. http://dx.doi.org/10.1002/zamm.19790590910

[12] Sakiadis, B.C. (1961) Boundary Layer Behavior on Continuous Solid Surfaces. AIChE Journal, 7, 26-28. http://dx.doi.org/10.1002/aic.690070108

[13] Liao, S.J. (2003) Beyond Perturbation: Introduction to Homotopy Analysis Method. Chapman and Hall, CRC Press, Florida. http://dx.doi.org/10.1201/9780203491164

[14] Liao, S.J. (1992) The Proposed Homotopy Analysis Technique for the Solution of Nonlinear Problem. Ph.D. Thesis, Shanghai Jiao Tong University, Shanghai.

[15] Liao, S.J. (2012) Homotopy Analysis Method in Nonlinear Differential Equations. Springer-Verlag Berlin Heidelberg. http://dx.doi.org/10.1007/978-3-642-25132-0

[16] Liao, S.J. (2013) Advances in the Homotopy Analysis Method. World Scientific Publishing Company, Singapore.

[17] Liao, S.J. (2009) Notes on the Homotopy Analysis Method: Some Definitions and Theorems. Communications in Nonlinear Science and Numerical Simulation, 14, 983-997.

[18] Ayub, M., Zaman, H., Sajid, M. and Hayat, T. (2008) Analytical Solution of Stagnation-Point Flow of a Viscoelastic Fluid towards a Stretching Surface. Communications in Nonlinear Science and Numerical Simulation, 13, 1822-1835. http://dx.doi.org/10.1016/j.cnsns.2007.04.021

[19] Zaman, H. and Ayub, M. (2010) Series Solution of Unsteady Free Convection Flow with Mass Transfer along an Accelerated Vertical Porous Plate with Suction. Central European Journal of Physics, 8, 931-939. http://dx.doi.org/10.2478/s11534-010-0007-y

[20] Hady, F.M. and Gorla, R.S.R. (1998) Heat Transfer from a Continuous Surface in a Parallel Free Stream of Viscoelastic Fluid. Acta Mechanica, 128, 201-208. http://dx.doi.org/10.1007/BF01251890

[21] Zaman, H., Hayat, T., Ayub, M. and Gorla, R.S.R. (2011) Series Solution for Heat Transfer from a Continuous Surface in a Parallel Free Stream of Viscoelastic Fluid. Numerical Methods for Partial Differential Equations, 27, 1511-1524. http://dx.doi.org/10.1002/num.20593 